\title{
Gain-of-function mutations in the phosphatidylserine synthase 1 (PTDSS1) gene cause Lenz-Majewski syndrome
}

\author{
Sérgio B Sousa ${ }^{1,2}$, Dagan Jenkins ${ }^{3,18}$, Estelle Chanudet ${ }^{4,18}$, Guergana Tasseva ${ }^{5,18}$, Miho Ishida ${ }^{1}$, Glenn Anderson ${ }^{6}$, \\ James Docker ${ }^{7}$, Mina Ryten ${ }^{8,9}$, Joaquim Sa ${ }^{2}$, Jorge M Saraiva ${ }^{2,10}$, Angela Barnicoat ${ }^{11}$, Richard Scott ${ }^{11}$, \\ Alistair Calder ${ }^{12}$, Duangrurdee Wattanasirichaigoon ${ }^{13}$, Krystyna Chrzanowska ${ }^{14}$, Martina Simandlová ${ }^{15}$, \\ Lionel Van Maldergem ${ }^{16,17}$, Philip Stanier ${ }^{7}$, Philip L Beales ${ }^{3,4}$, Jean E Vance ${ }^{5}$ \& Gudrun E Moore ${ }^{1}$
}

\begin{abstract}
Lenz-Majewski syndrome (LMS) is a syndrome of intellectual disability and multiple congenital anomalies that features generalized craniotubular hyperostosis. By using whole-exome sequencing and selecting variants consistent with the predicted dominant de novo etiology of LMS, we identified causative heterozygous missense mutations in PTDSS1, which encodes phosphatidylserine synthase 1 (PSS1). PSS1 is one of two enzymes involved in the production of phosphatidylserine. Phosphatidylserine synthesis was increased in intact fibroblasts from affected individuals, and end-product inhibition of PSS1 by phosphatidylserine was markedly reduced. Therefore, these mutations cause a gain-of-function effect associated with regulatory dysfunction of PSS1. We have identified LMS as the first human disease, to our knowledge, caused by disrupted phosphatidylserine metabolism. Our results point to an unexplored link between phosphatidylserine synthesis and bone metabolism.
\end{abstract}

LMS (MIM 151050), also known as Lenz-Majewski hyperostotic dwarfism, is recognized by the combination of sclerosing bone dysplasia, intellectual disability and distinct craniofacial, dental, cutaneous and distal-limb anomalies. LMS constitutes a unique and well-defined but very rare condition, with a total of eight typical cases reported so far ${ }^{1-10}$. The progressive generalized hyperostosis associated with this syndrome affects the cranium, the vertebrae and the diaphyses of tubular bones, leading to severe growth restriction. All known affected individuals are isolated cases from various ancestry groups, with no difference in severity or prevalence attributable to the sex of the affected individual. Neither familial recurrence nor consanguinity has been observed for any case. These characteristics and the very specific, consistent phenotype led to the hypothesis that LMS might be caused by de novo heterozygous dominant mutations in a single gene.

We collected DNA and clinical data from five unrelated LMSaffected individuals, including two who were new to this study (subjects 1 and 5) and three who were previously described (subjects $2-4)^{8-10}$ (Fig. 1 and Supplementary Table 1). No pathogenic copy number variants were detected using karyotype analysis (all cases) or array comparative genomic hybridization (NimbleGen 135k) (subject 1). To identify the underlying genetic cause of LMS, we performed whole-exome sequencing in subjects 1-4 and in both parents of subjects 1 and 4 (Supplementary Table 2). A total of 224 genes showed at least 1 rare or previously unreported variant in 2 or more affected individuals, of which only 11 were predicted to be functionally impaired by variants fitting the predicted mode of inheritance (Supplementary Table 3). A single gene, PTDSS1 (encoding phosphatidylserine synthase 1), contained putative mutations in all affected individuals. Three individuals (subjects 2-4) harbored an identical heterozygous missense c.1058A $>$ G mutation encoding a p.Gln $353 \mathrm{Arg}$ alteration. Subject 1 had a different heterozygous missense mutation, c. $805 \mathrm{C}>\mathrm{T}$, encoding a p.Pro269Ser alteration. Subject 5 was a previously unreported case in whom Sanger sequencing of the PTDSS1 coding region identified a third heterozygous mutation, c.794T $>C$, encoding a p.Leu265Pro alteration. All variants were absent from our private exome sequencing database of phenotypically characterized samples and had not been reported in public databases ${ }^{11,12}$. Each mutation was validated by Sanger sequencing (Supplementary Fig. 1) and was confirmed to be de novo in the four subjects for whom

\footnotetext{
${ }^{1}$ Clinical and Molecular Genetics Unit, University College London (UCL) Institute of Child Health, London, UK. 2Serviço de Genética Médica, Hospital Pediátrico, Centro Hospitalar e Universitário de Coimbra, Coimbra, Portugal. ${ }^{3}$ Molecular Medicine Unit, UCL Institute of Child Health, London, UK. ${ }^{4}$ Centre for Translational Genomics-GOSgene, UCL Institute of Child Health, London, UK. ${ }^{5}$ Group on the Molecular and Cell Biology of Lipids, Department of Medicine, University of Alberta, Edmonton, Alberta, Canada. ${ }^{6}$ Histopathology Department, Great Ormond Street Hospital for Children, London, UK. ${ }^{7}$ Neural Development Unit, UCL Institute of Child

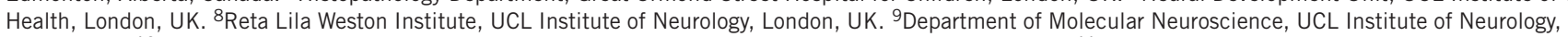
London, UK. ${ }^{10}$ University Clinic of Pediatrics, Faculty of Medicine, University of Coimbra, Coimbra, Portugal. ${ }^{11}$ Clinical Genetics Department, Great Ormond Street Hospital, London, UK. ${ }^{12}$ Radiology Department, Great Ormond Street Hospital, London, UK. ${ }^{13}$ Department of Pediatrics, Faculty of Medicine, Ramathibodi Hospital, Mahidol University, Bangkok, Thailand. ${ }^{14}$ Department of Medical Genetics, Children's Memorial Health Institute, Warsaw, Poland. ${ }^{15}$ Department of Biology and Medical Genetics, University Hospital Motol and Second Faculty of Medicine, Prague, Czech Republic. ${ }^{16}$ Centre de Génétique Humaine, Université de Franche-Comté, Besançon, France. ${ }^{17}$ Cutis Laxa Study Group, University of Franche-Comté, Besancon, France. ${ }^{18}$ These authors contributed equally to this work. Correspondence should be addressed to S.B.S. (sbsousa@chc.min-saude.pt).
} 
Figure 1 Clinical and radiological manifestations of individuals with LMS. (a) Photographs of subject 2 at 7 years (top left panel) and 17 years (other panels), after total dental removal and tongue reduction surgery. (b) Photographs of subject 4 at 36 years. (c) Radiographs of subject 1 at 4 months. (d) Radiographs of subject 3 at 10 years. (e) Photographs and radiographs of subject 5 at 7 years. Note the typical craniofacial dysmorphic features (broad prominent forehead, hypertelorism, long philtrum, prognathism), wide persistent fontanel, thickness of the jaw, clavicles and ribs, and distal-limb abnormalities (brachydactyly with proximal symphalangism and progressive shortening and/or fusion of the fourth and fifth rays). Cutis laxa is most evident in subject 5. Phenotypic expression is variable: subjects 2 and 4 harbor the same PTDSS 1 mutation, and subjects 2-4 were previously described, with progression of phenotypes apparent through comparison with the original report ${ }^{8-10}$. Informed consent was obtained for publication of the facial photographs shown.

parental DNA was available. All mutations occurred at highly conserved residues (Supplementary Fig. 2), affected all proteincoding transcripts of PTDSS1 and were predicted to be deleterious by all in silico tools used (Supplementary Table 4).

Phosphatidylserine is produced in mammalian cells by two synthases-phosphatidylserine synthase 1 (PSS1), encoded by PTDSS1, and phosphatidylserine synthase 2 (PSS2), encoded by PTDSS2. PSS1 synthesizes phosphatidylserine through the exchange of L-serine with the choline moiety of phosphatidylcholine $\mathrm{e}^{13,14}$ (Fig. 2a). In contrast, PSS2 converts phosphatidylethanolamine into phosphatidylserine by a parallel base-exchange reaction ${ }^{13,14}$. Phosphatidylserine represents $3-10 \%$ of all membrane phospholipids in mammalian tissues and cultured cells ${ }^{14}$. The relatively low abundance of phosphatidylserine is outweighed by its physiological importance, attributable to its unique physical and biochemical properties ${ }^{15}$ and its specific tissue $^{13,16}$ and subcellular distributions ${ }^{13,17}$. Functions of phosphatidylserine at the cell surface, including roles in apoptosis, coagulation and the internalization of viruses, have been studied extensively, but phosphatidylserine also participates in intracellular processes by interacting with key signaling proteins such as the Ras and Rho family of GTPases and protein kinase $\mathrm{C}^{13}$.

The amino acid sequences of PSS1 and PSS2 are $30 \%$ identical $^{18,19}$, and the residues involved in the identified alterations in PSS1 are common to PSS2. Applying alanine-scanning mutagenesis of a Chinese hamster PSS1 clone, Ohsawa et al. ${ }^{20}$ described three groups of mutations that differentially affected PSS1 activity, protein levels and regulation (Fig. 2b). The mutations resulting in p.Leu265Pro and p.Pro269Ser are adjacent to two mutations that increase PSS1 activity and attenuate negative feedback control of PSS1 by phosphatidylserine (Fig. 2b). Mice lacking either Ptdss1 or Ptdss 2 are viable, phenotypically normal and fertile, indicating that, in mice, PSS1 and PSS2 can compensate for each other 21,22 . In contrast, simultaneous disruption of both genes is lethal, implying that phosphatidylserine is absolutely required for viability ${ }^{21}$. In exome data from the four LMS cases analyzed, there was good coverage of PTDSS2, and no previously unreported variants were identified.
The absence of an overtly aberrant phenotype in Ptdss 1 knockout mice $^{21,22}$ and the positions of the PTDSS1 missense mutations identified in our subjects with LMS suggest that these mutations cause a gain of function, possibly associated with regulatory dysfunction. This reasoning is supported by the description of at least two individuals with large $8 \mathrm{q} 22.1$ deletions encompassing PTDSS1 that do not have features of $\mathrm{LMS}^{23}$. We therefore performed enzymatic studies in skin fibroblasts from subjects 1, 4 and 5 who harbored p.Pro269Ser, p.Gln353Arg and p.Leu265Pro alterations, respectively. The in vitro serine-exchange activity of PSS1 was not notably higher in fibroblasts from the affected subjects than in control fibroblasts (Fig. 3a,b), and PSS2 activity was similar in both groups (Fig. 3a). We further investigated the synthesis of phosphatidylserine and phosphatidylserine-derived phosphatidylethanolamine in radiolabeling experiments with $\left[{ }^{3} \mathrm{H}\right]$ serine in intact fibroblasts from subjects with LMS and control individuals. The incorporation of radiolabel into phosphatidylserine and phosphatidylserine-derived phosphatidylethanolamine was markedly higher in cells derived from individuals with LMS than in control cells (Fig. 3c,d), demonstrating that these PTDSS1 mutations profoundly increase phosphatidylserine synthesis in intact fibroblasts.

We next investigated the reason why alterations in PSS1 resulted in increased phosphatidylserine synthesis in fibroblasts from individuals with LMS. We first performed reverse-transcription quantitative PCR (RT-qPCR) analysis of PTDSS1 and PTDSS2 in fibroblasts from control individuals and those with LMS and observed similar mRNA levels in the two groups (Fig. 4a,b). Moreover, immunoblotting showed that the amount of PSS1 protein was not higher in fibroblasts from subjects with LMS than in controls (Fig. 4c). Thus, the higher rate of synthesis of phosphatidylserine and phosphatidylethanolamine in the mutant fibroblasts (Fig. 3c,d) was not due to increased levels of PSS1, nor were any compensatory changes detected in the levels of PTDSS2 mRNA (Fig. $\mathbf{4 b}$ ) or PSS2 activity (Fig. 3a). To determine 


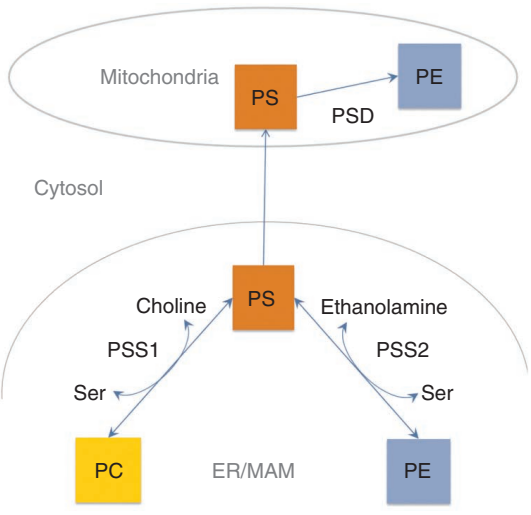

b

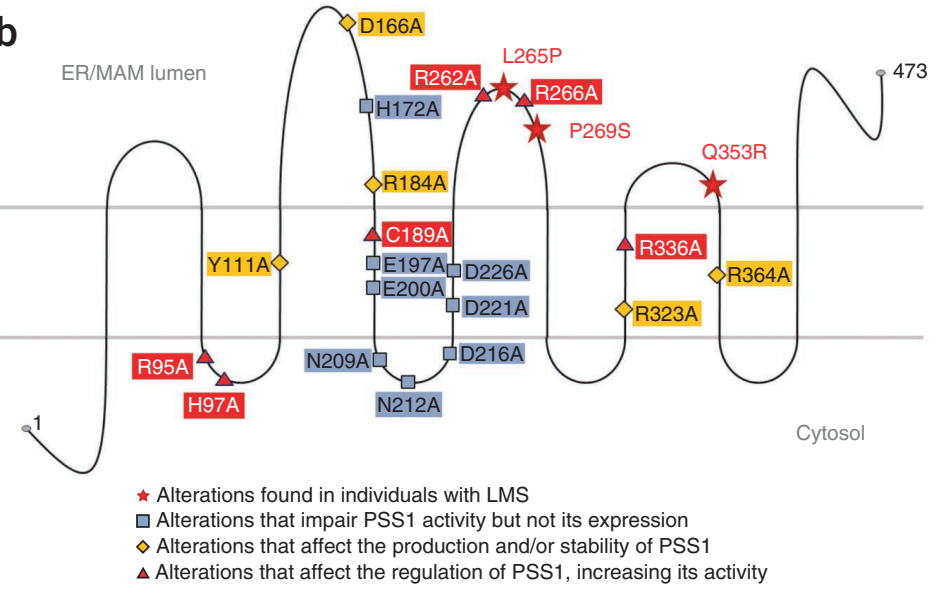

Figure 2 Overview of phosphatidylserine metabolism and the PSS1 protein. (a) Subcellular location of phosphatidylserine (PS) pathway components. In mammalian cells, phosphatidylserine is made in the endoplasmic reticulum/mitochondria-associated membranes (ER/MAM) 45 by exchange of serine for the choline or ethanolamine head groups of phosphatidylcholine (PC) or phosphatidylethanolamine (PE) by PSS1 and PSS2, respectively. Newly synthesized phosphatidylserine is transferred via MAM to the mitochondria, where phosphatidylserine is decarboxylated to phosphatidylethanolamine by phosphatidylserine decarboxylase (PSD) on the mitochondrial inner membrane. (b) Topology of the PSS1 protein and alterations identified in individuals with LMS. PSS1 is predicted to have nine transmembrane domains according to UniProt (P48651). Locations of the alterations in LMS are compared with the alterations induced by alanine-scanning mutagenesis ${ }^{20}$. The alterations in LMS are located in non-transmembrane domains in the ER/MAM lumen. p.Leu265Pro and p.Pro269Ser lie adjacent to the p.Arg262Ala and p.Arg266Ala alterations that increase PSS1 activity and are resistant to inhibition by phosphatidylserine, a process that requires Arg95 of PSS1 and Arg97 of PSS2 in CHO cells ${ }^{24-26 .}$

whether these mutations led to the impairment of end-product inhibition of PSS1, we compared phosphatidylserine synthesis in control and mutant fibroblasts in the presence and absence of exogenous phosphatidylserine $e^{20,24-26}$. In contrast to control cells, intact fibroblasts from individuals with LMS showed incorporation of $\left[{ }^{3} \mathrm{H}\right]$ serine into phosphatidylserine and phosphatidylethanolamine that was profoundly resistant to inhibition by phosphatidylserine (Fig. 4d,e). This inhibition was specific, as addition of phosphatidylcholine instead of phosphatidylserine did not reduce the rate of phosphatidylserine synthesis (Supplementary Fig. 3). The in vitro enzymatic activity of phosphatidylserine synthase in lysates from control but not mutant fibroblasts was also strongly inhibited by the addition of phosphatidylserine (Supplementary Fig. 4 and Supplementary Data Set). However, despite the marked disturbance in phosphatidylserine synthesis in fibroblasts from individuals with LMS, the amounts of cellular phosphatidylserine, phosphatidylethanolamine and phosphatidylcholine (Fig. 4f) remained unaltered relative to control cells.
Although one might anticipate that an increase in phosphatidylserine synthesis in the mutant cells would increase the steady-state levels of cellular phosphatidylserine, cellular phospholipid homeostasis is tightly regulated with respect to both synthesis and degradation. For example, PSS1 overexpression in HEK293 cells only slightly increases cellular phosphatidylserine levels ${ }^{27}$. Furthermore, overexpression of PSS1 or PSS2 in McArdle hepatoma cells does not increase cellular phosphatidylserine or phosphatidylethanolamine content ${ }^{19,28}$. In addition, in the majority of PSS1-deficient ${ }^{21}$ or PSS2-deficient ${ }^{22}$ mouse tissues (including the brain), the levels of phosphatidylserine and phosphatidylethanolamine are not reduced, despite the $65-98 \%$ reduction in total serine-exchange activity ${ }^{21}$. Nevertheless, it is possible that, in individuals with LMS, the phospholipid content of specific tissues and subcellular organelles is altered (particularly with regard to phosphatidylserine and the distribution of its subclasses) so that only signaling processes important in the development and function of affected tissues are disturbed. Alternatively, the mutations in LMS a

$\square$ No addition $\square 50 \mathrm{mM}$ choline

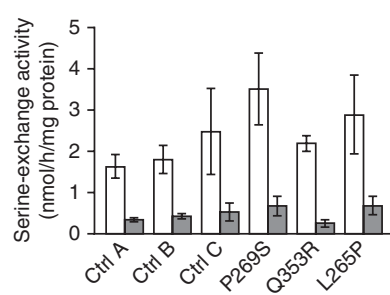

b

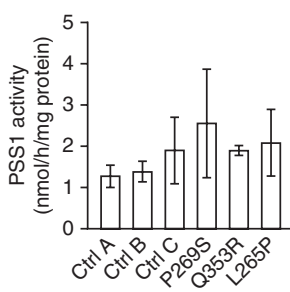

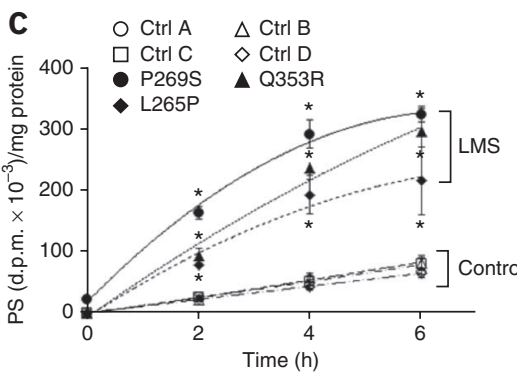

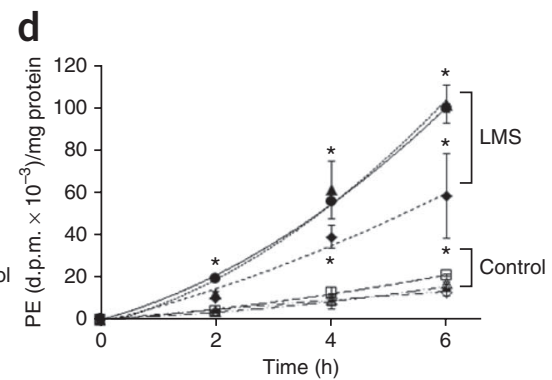

Figure 3 Phosphatidylserine synthesis is increased in cultured fibroblasts from individuals with LMS compared to control fibroblasts. (a) In vitro serine-exchange activity was measured in the absence (no addition, white; represents activity of PSS1 and PSS2) and presence (gray; PSS2 activity alone) of $50 \mathrm{mM}$ choline in lysates of fibroblasts from three controls (Ctrl A, B and C) and three individuals with LMS (subject 1, p.Pro269Ser; subject 4, p.GIn353Arg; subject 5, p.Leu265Pro). (b) PSS1 activity was calculated by subtracting activity in the presence of 50 mM choline from total activity (no addition). Data in a,b are the mean values \pm s.d. from three independent experiments, each performed in triplicate. (c, d) Fibroblasts from four controls (Ctrl A, B, C and D) and three individuals with LMS were incubated with $\left[{ }^{3} \mathrm{H}\right.$ ] serine for $\mathrm{O}$ to $6 \mathrm{~h}$, and incorporation of radioactivity into phosphatidylserine (c) and phosphatidylethanolamine (d) was quantified. Data in c,d are the mean values \pm s.d. from triplicate analyses from each of two experiments. ${ }^{*} P<0.05$ at 2,4 and 6 h for LMS versus control cells. Some error bars are too small to be visible. 

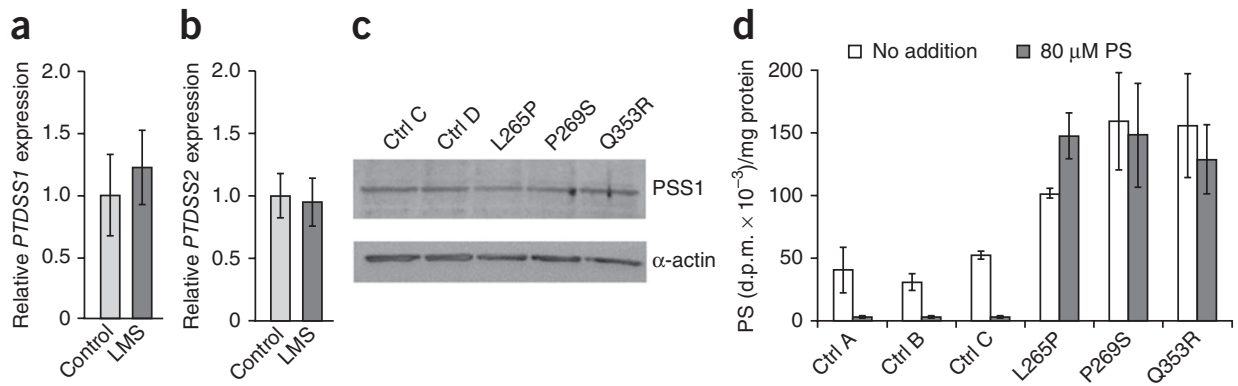

Figure 4 Phosphatidylserine synthase activity in fibroblasts from individuals with LMS is resistant to inhibition by phosphatidylserine, whereas PSS1 mRNA and protein expression, PSS2 mRNA expression and total cellular aminophospholipid content are normal. (a,b) RT-qPCR analysis of fibroblasts from individuals with LMS and controls for PTDSS1 (a) and PTDSS2 (b). Data were generated from duplicate RNA samples from each of the three subjects and six controls. mRNA levels were quantified using GAPDH mRNA levels as an endogenous control. Data in $\mathbf{a}, \mathbf{b}$ are mean values \pm s.e.m. and are presented relative to the average value for the controls. (c) PSS1 immunoblot in fibroblasts from two control individuals and three individuals with LMS, representative of two independent blots performed with different samples, and using $\alpha$-actin as a loading control. $(\mathbf{d}, \mathrm{e})\left[{ }^{3} \mathrm{H}\right]$ serine labeling of intact fibroblasts in the absence (no addition) or presence $(80 \mu \mathrm{M})$ of phosphatidylserine liposomes. Incorporation of radioactivity into phosphatidylserine (d) and phosphatidylserine-derived phosphatidylethanolamine (e) was analyzed. (f) Phospholipid mass of phosphatidylcholine, phosphatidylethanolamine and phosphatidylserine in the lysates of skin fibroblasts from three individuals with LMS (subjects 1 , 4 and 5) and three controls (Ctrl A, B and C). $P_{i}$, inorganic phosphate. Mean values $\pm s . d$. in $\mathbf{d}-\mathbf{f}$ are from triplicate analyses of one of three independent experiments with similar results. For $\mathbf{f}$, LMS versus control $P>0.05$. might disrupt an unknown function of PSS1 or lead to altered substrate specificity and/or gain of a new function.

We examined the developmental consequences of the mutations identified in LMS and the resulting increases in in vivo PSS1 activity by expressing varying doses of RNA encoding the wild-type and mutant forms of human PTDSS1 in zebrafish embryos (Fig. 5). Injection of embryos with up to $750 \mathrm{pg}$ (the highest non-toxic dose) of wild-type PTDSS1 RNA at the one-cell stage did not cause any embryonic defects (Fig. 5a,d,e,h and data not shown). In contrast, injection of embryos with RNA encoding the mutant forms of PSS1 identified in individuals with LMS caused a variety of defects in $6-40 \%$ of embryos $5 \mathrm{~d}$ after fertilization, even at the lowest dose tested (100 pg). Defects included craniofacial anomalies that resembled the ones in individuals with LMS (Figs. 1 and 5d-g). Similar defects occurred in embryos injected with RNA encoding all three missense mutations, and the frequency of the defects was dose dependent (Fig. 5h). In each case, these findings support a gain-of-function effect of the PTDSS1 mutation. Craniofacial defects were not rescued upon coinjection with tp53 morpholino (Fig. 5h), a well-studied apoptotic inhibitor ${ }^{29}$, which we confirmed by TUNEL staining inhibited apoptosis in developing embryos (data not shown). Therefore, the mechanisms underlying these defects are not likely to be caused by enhanced p53-dependent apoptotic cell death. Indeed, no gross differences in apoptosis or cell proliferation were observed in the craniofacial region of embryos overexpressing either wild-type or mutant PTDSS1 (Supplementary Figs. 5 and 6). In contrast, patterning of neural crest cells within the craniofacial cartilage of embryos injected with PTDSS1 RNA encoding p.Pro269Leu was abnormal (Fig. 5i-1). The frequency of these defects was somewhat higher with RNA encoding p.Leu265Pro and p.Pro269Leu. These alterations cluster with previously studied activating mutations (Fig. 2b), unlike the p.Gln353Arg alteration, which resides in a separate cytosolic domain. This observation might be consistent with a stronger gainof-function effect for the p.Leu265Pro and p.Pro269Leu alterations. Nevertheless, the biochemical data (Figs. 3 and 4) do not convincingly support this hypothesis, and no genotype-phenotype clinical correlations were evident (Supplementary Table 1).
RT-qPCR showed that PTDSS1 expression was enriched in the brain and skin, as has also been observed for the corresponding genes in mouse tissues ${ }^{30,31}$. Expression of PTDSS1 in bone was less pronounced than in the brain (Supplementary Fig. 7 and Supplementary Data Set). We also analyzed data from two independent studies of array-based expression in human brain RNA derived from large numbers of samples (Supplementary Fig. 8). PTDSS1 expression was highest in almost all areas of the brain and throughout fetal and postnatal life, with peak expression occurring during mid-gestation. Consistent with these observations, phosphatidylserine is important for brain function and development, and levels of phosphatidylserine and phosphatidylethanolamine are particularly high in mammalian brains ${ }^{16}$. Indeed, dietary supplementation with phosphatidylserine is widely, albeit controversially, used for improving memory, cognitive function and exercise performance ${ }^{32,33}$. It will therefore be important to understand how phosphatidylserine metabolism is disturbed in the nervous system of individuals with LMS and the specific pathogenic mechanisms that lead to their intellectual disability.

Individuals with LMS have abnormally loose skin, consistent with the cutis laxa spectrum. Skin histology showed a notable reduction in the amount of elastic tissue in one individual with $\mathrm{LMS}^{3}$, a finding that was confirmed by electron microscopy of a skin biopsy from subject 4 (Supplementary Fig. 9). In electron microscopy studies, skin fibroblasts from subjects 1 and 4 showed a striking degree of cytoplasmic vacuolization (Supplementary Fig. 9). Some of these vacuoles appeared to contain stored material that we hypothesized could have had a lipid component. Nevertheless, we did not observe marked differences between control fibroblasts and those isolated from individuals with LMS in either neutral lipid accumulation in lipid droplets or in the levels of triacylglycerols (Supplementary Fig. 10 and Supplementary Data Set).

An increasing number of metabolic disorders have been found to be associated with cutis laxa ${ }^{34}$. For example, autosomal recessive ATP6V0A2 mutations are associated with a phenotypic spectrum that overlaps that of LMS with respect to cutis laxa, intellectual disability, some dysmorphic features and large and late-closing anterior fontanel. 
Figure 5 Overexpression of mutant PTDSS1 RNA in zebrafish embryos disrupts normal development. (a-h) Zebrafish embryos injected with wild-type (WT) RNA $(\mathbf{a}, \mathbf{h})$ were normal at all doses tested, whereas embryos injected with mutant forms of RNA (p.Pro269Ser is shown) exhibited craniofacial defects, including small (b) or absent (c, arrow) eyes, severe angulation of the trunk (c, $\left.{ }^{*}\right)$ and abnormal cartilage shown by Alcian blue staining $(\mathbf{d}-\mathbf{g})$. (d,f) Ventral images. Note in the mutant embryo in $\mathbf{f}$ the widely spaced eyes, the short jaw and the wide angle of Meckel's cartilage. $(\mathbf{e}, \mathbf{g})$ Lateral images. (h) Proportion of embryos showing these defects for different doses of wild-type or mutant RNA (p.Gln353Arg, p.Pro269Ser or p.Leu265Pro). Embryos injected with wildtype RNA were unaffected, and the frequency with which embryos injected with mutant forms of PTDSS1 showed abnormalities was dose dependent. The proportion of embryos injected with RNA encoding p.Pro269Ser showing craniofacial defects was unaffected by coinjection with $p 53$ morpholino (MO; last column). NS, not significant. (i-I) Ventral

images of heterozygous sox10:gfp transgenic zebrafish embryos labeling neural crest derivatives with GFP. Note abnormal patterning of neural crest pharangeal arch-derived structures after injection with mutant PTDSS1 RNA (i,k), mirroring the craniofacial cartilage defects shown by Alcian blue staining $(\mathbf{d}, \mathbf{f})$. (j, I) Heat-map images of the intensity of GFP epifluorescence for the images in $\mathbf{i}$ and $\mathbf{k}$, showing a reduced contribution of neural crest cells within the craniofacial cartilage. Scale bars: $500 \mu \mathrm{m}$ in a-c; $200 \mu \mathrm{m}$ in d,f; $300 \mu \mathrm{m}$ in e,g; $200 \mu \mathrm{m}$ in $\mathbf{i}-\mathbf{I}$.

Individuals with ATP6VOA2 mutations have combined $\mathrm{N}$ - and $\mathrm{O}$ linked congenital glycosylation defects usually identified by a type 2 pattern on transferrin isoelectrofocusing ${ }^{35,36}$, analysis of which was normal in subject 5. Autosomal recessive mutations in PYCR1 (encoding pyrroline-5-carboxylate reductase 1 ) were found to be associated with various cutis laxa syndrome phenotypes ${ }^{35}$ that share intellectual disability and brain anomalies with LMS. Sequencing of the ATP6VOA2 and PYCR1 genes in subject 5 did not identify any variants. Because phosphatidylserine in the plasma membrane has a well-known role in the elimination of apoptotic cells by phagocytosis ${ }^{13}$ and because both ATP6VOA2 and PYCR1 mutations influence apoptosis ${ }^{35,36}$, one could hypothesize that this would also happen with PTDSS1 mutations. Nevertheless, disturbance to normal apoptosis was not evident in the zebrafish LMS model (Fig. 5h-1 and Supplementary Fig. 4) or in the electron microscopy studies (Supplementary Fig. 9), although we observed a significant increase in the percentage of Annexin Vstained cells in cultured fibroblasts from individuals with LMS compared to control cells (Supplementary Fig. 11 and Supplementary Data Set). In contrast to mutations in LMS, ATP6V0A2 and PYCR1 mutations are associated with decreased bone mineral density ${ }^{35,37}$.

No direct link has been established between phosphatidylserine metabolism and known pathways involved in skeletal dysplasias with increased bone density (especially the ones classified with LMS in group 24) ${ }^{38}$. Nevertheless, phosphatidylserine and its calcium-binding properties have been recognized as being important in bone mineralization, physiological and pathological calcification and dentine formation 39,40 . These processes could well be affected by changes in phosphatidylserine metabolism in LMS, as they involve the rearrangement of apoptotic cell membranes and the release of phosphatidylserineenriched vesicles ${ }^{40,41}$, and are tightly regulated by the osteoblast, the chondrocyte and the odontoblast lineages, cells similar to the fibroblasts studied here. Phosphatidylserine and phosphatidylserinemimicking coating technology promote calcification and have been explored as potential clinical bone repair applications for osteointe- grative biomaterials ${ }^{40}$. In other related skeletal dysplasias, such as Ghosal syndrome ${ }^{42}$ and craniometaphyseal dysplasia ${ }^{43}$, abnormal osteoblast-osteoclast communication seems to be the basis of the pathomechanism, which might also apply in LMS. Indeed, phosphatidylserine-containing liposomes were shown to inhibit osteoclast differentiation and prevent trabecular bone loss ${ }^{44}$, suggesting application of phosphatidylserine as a potential pharmacological intervention in osteoporosis. We propose, therefore, that osteoclast dysfunction, usually associated with osteopetrosis, might also be implicated in the generalized bone hyperostosis and sclerosis in individuals with LMS.

In summary, we have identified de novo heterozygous PTDSS1 missense mutations as the cause of LMS, the first human genetic disease, to our knowledge, to be associated with disturbance of phosphatidylserine metabolism. Studies of fibroblasts from affected individuals demonstrate that these mutations increase the rate of phosphatidylserine synthesis and profoundly impair negative feedback regulation of PSS1 activity by phosphatidylserine. Furthermore, LMS constitutes a rare example of a disease caused by dominant heterozygous gain-offunction mutations affecting an enzyme. It will therefore be important to better understand its pathogenicity by elucidating the contextdependent mechanisms and tissue- and subcellular location-specific consequences of increased phosphatidylserine synthesis, particularly in the brain and in bone metabolism. Such research will bring valuable insights into the pathogenesis of and potential treatments for common disorders that share features with LMS, particularly intellectual disabilities and the osteosclerosis/osteoporosis spectrum of conditions.

URLs. Exome Variant Server, National Heart, Lung, and Blood Institute (NHLBI) GO Exome Sequencing Project (ESP), http://evs. gs.washington.edu/EVS/; UCSC Genome Browser, http://genome. ucsc.edu/; Universal Protein Resource (UniProt), http://www.uniprot. org/; Burrows-Wheeler Aligner (BWA) version 0.5.9c, http://bio-bwa. sourceforge.net/; Picard version 1.45, http://picard.sourceforge.net/; 
ANNOVAR release 20 November 2011, http://www.openbioinformatics. org/annovar/; NCBI dbSNP Build 132, http://www.ncbi.nlm.nih. gov/projects/SNP/; 1000 Genomes Project, http://www.1000genomes. org/; Integrative Genomics Viewer (IGV), http://www.broadinstitute. org/igv/UserGuide; Ensembl Variant Effect Predictor, http://www. ensembl.org/info/docs/variation/vep/index.html; Human Brain Transcriptome, http://hbatlas.org/.

\section{METHODS}

Methods and any associated references are available in the online version of the paper.

Accession codes. Reference sequences for PTDSS1 are available from NCBI under accessions NM_014754.1 and NP_055569.1. Additional information on the PSS1 protein is available from UniProtKB under accession $\mathrm{P} 48651$.

Note: Any Supplementary Information and Source Data files are available in the online version of the paper.

\section{ACKNOWLEDGMENTS}

We are grateful to the individuals with LMS and their families for their participation in this study. We thank P. Clayton and R. Hennekam for their comments on the manuscript, A. Offiah for confirming the radiological diagnosis in subject 1 and W. Emmett for his valuable help in processing raw sequencing data. The authors would like to acknowledge D. Trabzuni and the UK Brain Expression Consortium, the Human Brain Transcriptome project at the Department of Neurobiology at the Yale University School of Medicine NHLBI for the use of data from their public database and the GO Exome Sequencing Project and its ongoing studies, which produced and provided exome variant calls for comparison, including the Lung GO Sequencing Project (HL-102923), the Women's Health Initiative (WHI) Sequencing Project (HL-102924), the Broad GO Sequencing Project (HL-102925), the Seattle GO Sequencing Project (HL-102926) and the Heart GO Sequencing Project (HL-103010). S.B.S. was supported by the Fundação para a Ciência e Tecnologia (SFRH/BD/46778/2008). The Centre for Translational Genomics-GOSgene is supported by the Biomedical Research Centre of the National Institute for Health Research at Great Ormond Street Hospital for Children NHS Foundation Trust and UCL Institute of Child Health. D.J. was supported by a Wellcome Trust ViP Award. D.W. is a recipient of Research Career Development Awards from the Faculty of Medicine at Ramathibodi Hospital. M.S. is supported by Czech research development grant 00064203. P.S. is supported by the Great Ormond Street Hospital Children's Charity (GOSHCC). The Fetal Growth and Development research team of G.E.M. is funded by WellBeing of Women, the Wellcome Trust, GOSHCC and the UK Medical Research Council. The research of J.E.V. is supported by the National Science and Engineering Council of Canada.

\section{AUTHOR CONTRIBUTIONS}

S.B.S. and G.E.M. designed the study. S.B.S., A.B., R.S., A.C., J.S., J.M.S., D.W., K.C., M.S. and L.V.M. characterized individuals with LMS and collected clinical data and samples. S.B.S. designed and performed direct mutagenesis, zebrafish experiments, Sanger sequencing and cell culture and wrote the manuscript. D.J. designed and performed zebrafish experiments and wrote the manuscript. E.C. designed and performed next-generation sequencing data analysis and segregation studies and wrote the manuscript. J.E.V. and G.T. designed and performed phospholipid metabolism studies and wrote the manuscript. M.I. performed RT-qPCR experiments. G.A. performed electron microscopy studies. M.R. contributed data for human adult brain transcriptome analysis. J.D. and P.S. performed Annexin V staining experiments. P.S. and P.L.B. contributed to experimental design and data analysis. All authors discussed the results and implications of the work and commented on the manuscript.

\section{COMPETING FINANCIAL INTERESTS}

The authors declare no competing financial interests.

Reprints and permissions information is available online at http://www.nature.com/ reprints/index.html.

1. Braham, R.L. Multiple congenital abnormalities with diaphyseal dysplasia (Ca murati-Engelmann's syndrome). Report of a case. Oral Surg. Oral Med. Oral Pathol. 27, 20-26 (1969).
2. Macpherson, R.I. Craniodiaphyseal dysplasia, a disease or group of diseases? J. Can. Assoc. Radiol. 25, 22-33 (1974).

3. Kaye, C.I., Fisher, D.E. \& Esterly, N.B. Cutis laxa, skeletal anomalies, and ambiguous genitalia. Am. J. Dis. Child. 127, 115-117 (1974)

4. Lenz, W.D. \& Majewski, F. A generalized disorders of the connective tissues with progeria, choanal atresia, symphalangism, hypoplasia of dentine and craniodiaphyseal hypostosis. Birth Defects Orig. Artic. Ser. 10, 133-136 (1974).

5. Majewski, F. Lenz-Majewski hyperostotic dwarfism: reexamination of the original patient. Am. J. Med. Genet. 93, 335-338 (2000).

6. Robinow, M., Johanson, A.J. \& Smith, T.H. The Lenz-Majewski hyperostotic dwarfism. A syndrome of multiple congenital anomalies, mental retardation, and progressive skeletal sclerosis. J. Pediatr. 91, 417-421 (1977).

7. Gorlin, R.J. \& Whitley, C.B. Lenz-Majewski syndrome. Radiology 149, 129-131 (1983).

8. Chrzanowska, K.H., Fryns, J.P., Krajewska-Walasek, M., Van den Berghe, H. \& Wisniewski, L. Skeletal dysplasia syndrome with progeroid appearance, characteristic facial and limb anomalies, multiple synostoses, and distinct skeletal changes: a variant example of the Lenz-Majewski syndrome. Am. J. Med. Genet. 32, 470-474 (1989).

9. Saraiva, J.M. Dysgenesis of corpus callosum in Lenz-Majewski hyperostotic dwarfism. Am. J. Med. Genet. 91, 198-200 (2000).

10. Wattanasirichaigoon, D., Visudtibhan, A., Jaovisidha, S., Laothamatas, J. \& Chunharas, A. Expanding the phenotypic spectrum of Lenz-Majewski syndrome: facial palsy, cleft palate and hydrocephalus. Clin. Dysmorphol. 13, 137-142 (2004).

11. Sherry, S.T. et al. dbSNP: the NCBI database of genetic variation. Nucleic Acids Res. 29, 308-311 (2001)

12. 1000 Genomes Project Consortium. A map of human genome variation from population-scale sequencing. Nature 467, 1061-1073 (2010).

13. Vance, J.E. \& Tasseva, G. Formation and function of phosphatidylserine and phosphatidylethanolamine in mammalian cells. Biochim. Biophys. Acta 1831, 543-554 (2013).

14. Tomohiro, S., Kawaguti, A., Kawabe, Y., Kitada, S. \& Kuge, O. Purification and characterization of human phosphatidylserine synthases 1 and 2. Biochem. J. 418, 421-429 (2009)

15. Leventis, P.A. \& Grinstein, S. The distribution and function of phosphatidylserine in cellular membranes. Annu. Rev. Biophys. 39, 407-427 (2010).

16. Mozzi, R., Buratta, S. \& Goracci, G. Metabolism and functions of phosphatidylserine in mammalian brain. Neurochem. Res. 28, 195-214 (2003).

17. Schick, P.K., Kurica, K.B. \& Chacko, G.K. Location of phosphatidylethanolamine and phosphatidylserine in the human platelet plasma membrane. J. Clin. Invest. 57, 1221-1226 (1976)

18. Saito, K., Nishijima, M. \& Kuge, O. Genetic evidence that phosphatidylserine synthase II catalyses the conversion of phosphatidylethanolamine to phosphatidylserine in Chinese Hamster Ovary cells. Biochemistry 273, 17199-17205 (1998).

19. Stone, S.J. \& Vance, J.E. Cloning and expression of murine liver phosphatidylserine synthase (PSS)-2: differential regulation of phospholipid metabolism by PSS1 and PSS2. Biochem. J. 342, 57-64 (1999).

20. Ohsawa, T., Nishijima, M. \& Kuge, O. Functional analysis of Chinese hamster phosphatidylserine synthase 1 through systematic alanine mutagenesis. Biochem. J. 381, 853-859 (2004).

21. Arikketh, D., Nelson, R. \& Vance, J.E. Defining the importance of phosphatidylserine synthase-1 (PSS1): unexpected viability of PSS1-deficient mice. J. Biol. Chem. 283, 12888-12897 (2008)

22. Bergo, M.O. et al. Defining the importance of phosphatidylserine synthase 2 in mice. J. Biol. Chem. 277, 47701-47708 (2002)

23. Allanson, J. et al. Nablus mask-like facial syndrome: deletion of chromosome $8 q 22.1$ is necessary but not sufficient to cause the phenotype. Am. J. Med. Genet. A. 158A, 2091-2099 (2012).

24. Nishijimas, M., Kuge, O. \& Akamatsu, Y. Phosphatidylserine biosynthesis in cultured Chinese hamster ovary cells. I. Inhibition of de novo phosphatidylserine biosynthesis by exogenous phosphatidylserine and its efficient incorporation. J. Biol. Chem. 261, 5784-5789 (1986).

25. Kuge, O., Saito, K. \& Nishijima, M. Control of phosphatidylserine synthase II activity in Chinese hamster ovary cells. J. Biol. Chem. 274, 23844-23849 (1999).

26. Kuge, O., Hasegawa, K., Saito, K. \& Nishijima, M. Control of phosphatidylserine biosynthesis through phosphatidylserine-mediated inhibition of phosphatidylserine synthase 1 in Chinese hamster ovary cells. Proc. Natl. Acad. Sci. USA 95 4199-4203 (1998)

27. Morita, S.-Y. et al. Enzymatic measurement of phosphatidylserine in cultured cells. J. Lipid Res. 53, 325-330 (2012)

28. Stone, S.J., Cui, Z. \& Vance, J.E. Cloning and expression of mouse live phosphatidylserine synthase-1 cDNA. J. Biol. Chem. 273, 7293-7302 (1998).

29. Gerety, S.S. \& Wilkinson, D.G. Morpholino artifacts provide pitfalls and reveal a novel role for pro-apoptotic genes in hindbrain boundary development. Dev. Biol. 350, 279-289 (2011).

30. Sturbois-Balcerzak, B., Stone, S.J., Sreenivas, A. \& Vance, J.E. Structure and expression of the murine phosphatidylserine synthase-1 gene. J. Biol. Chem. 276 8205-8212 (2001).

31. Tasseva, G., Cole, L. \& Vance, J.E. N-Myc and SP regulate phosphatidylserine synthase-1 expression in brain and glial cells. J. Biol. Chem. 286, 1061-1073 (2011).

32. Kingsley, M. Effects of phosphatidylserine supplementation on exercising humans. Sports Med. 36, 657-669 (2006). 
33. Serby, M.J., Burns, S.J. \& Roane, D.M. Treatment of memory loss with herbal remedies. Curr. Treat. Options Neurol. 13, 520-528 (2011).

34. Mohamed, M. et al. Metabolic cutis laxa syndromes. J. Inherit. Metab. Dis. 34, 907-916 (2011).

35. Reversade, B. et al. Mutations in PYCR1 cause cutis laxa with progeroid features. Nat. Genet. 41, 1016-1021 (2009).

36. Hucthagowder, V. et al. Loss-of-function mutations in ATP6VOA2 impair vesicular trafficking, tropoelastin secretion and cell survival. Hum. Mol. Genet. 18, 2149-2165 (2009).

37. Fischer, B. et al. Further characterization of ATP6VOA2-related autosomal recessive cutis laxa. Hum. Genet. 131, 1761-1773 (2012).

38. Warman, M.L. et al. Nosology and classification of genetic skeletal disorders: 2010 revision. Am. J. Med. Genet. A. 155A, 943-968 (2011).

39. Wu, L.N.Y., Genge, B.R. \& Wuthier, R.E. Analysis and molecular modeling of the formation, structure, and activity of the phosphatidylserine-calcium-phosphate complex associated with biomineralization. J. Biol. Chem. 283, 3827-3838 (2008).
40. Merolli, A \& Santin, M. Role of phosphatidyl-serine in bone repair and its technological exploitation. Molecules 14, 5367-5381 (2009).

41. Thouverey, C., Strzelecka-Kiliszek, A., Balcerzak, M., Buchet, R. \& Pikula, S. Matrix vesicles originate from apical membrane microvilli of mineralizing osteoblast-like Saos-2 cells. J. Cell. Biochem. 106, 127-138 (2009).

42. Geneviève, D. et al. Thromboxane synthase mutations in an increased bone density disorder (Ghosal syndrome). Nat. Genet. 40, 284-286 (2008).

43. Chen, I.-P., Wang, L., Jiang, X., Aguila, H.L. \& Reichenberger, E.J.A. Phe377del mutation in ANK leads to impaired osteoblastogenesis and osteoclastogenesis in a mouse model for craniometaphyseal dysplasia (CMD). Hum. Mol. Genet. 20, 948-961 (2011).

44. Wu, Z., Ma, H.M., Kukita, T., Nakanishi, Y. \& Nakanishi, H. Phosphatidylserinecontaining liposomes inhibit the differentiation of osteoclasts and trabecular bone loss. J. Immunol. 184, 3191-3201 (2010).

45. Stone, S.J. \& Vance, J.E. Phosphatidylserine synthase- 1 and -2 are localized to mitochondria-associated membranes. J. Biol. Chem. 275, 34534-34540 (2000). 


\section{ONLINE METHODS}

Cases and samples. Samples were collected from five affected individuals with typical LMS from different ancestry groups (Supplementary Table 1). Phenotypic data were collected from a detailed review of medical records, phone interviews, clinical re-evaluation and photographs. All participants provided written informed consent, and the Institute of Child Health/Great Ormond Street Hospital Research Ethics Committee approved the study.

Exome capture and sequencing. Four affected individuals were screened by whole-exome sequencing, two as a trio including the proband and both parents. In each case, genomic DNA was enriched for exonic regions using the SureSelect All Exon 50Mb Targeted Enrichment kit (targeting 202,124 exons from 20,718 genes) from Agilent Technologies, according to the manufacturer's protocol. Captured libraries were sequenced on an Illumina HiSeq 2000 instrument using Illumina sBot clustering and HiSeq chemistries v1.0, under a paired-end 100-bp read-length protocol, with four samples per flow cell lane to achieve minimum median coverage of $60 x$.

Data processing. Reads were aligned to the human reference genome (GRCh37/hg19) using the Burrows-Wheeler Aligner (BWA version 0.5.9c). The GATK tool suite (GATK version v1.4-17-gd5199db) was used to call single-nucleotide variants and indels, following recommendations for singlesample calls. This analysis included preprocessing with marking of PCR duplicates using Picard (version 1.45), base quality score recalibration and local realignments. Variable quality score recalibration (VQSR) was applied using the following publicly available data sets: dbsnp_132.hg19.vcf, hapmap_3.3, 1000G_omni2.5 and Mills_Devine_2hit indel set. Variant annotation was carried out via ANNOVAR (release from 20 November 2011). Exome sequencing data are available upon request.

Data analyses and validation. In trios, variants were filtered according to a de novo mode of inheritance using an in-house script. Variants were prioritized according to frequency, predicted functional impact and occurrence in the four affected individuals, at both the sequence position and gene levels. Considering the rarity of the phenotype, we excluded variants if they were found in any sample of our in-house database containing $>80$ exomes from well-characterized individuals and controls that have been similarly processed. Rare and newly identified variants that were present in less than $0.5 \%$ of samples in the NCBI dbSNP Build 132 and 1000 Genomes Project databases and that affected the same gene in at least two of the four affected individuals were individually assessed. Mapping quality assessment included consideration of mapping score, coverage, bidirectional reads and genomic mappability (repeats and duplications) and visualization using the Integrative Genomics Viewer (IGV). Functional assessment included predictions from the Ensembl Variant Effect Predictor with SIFT and PolyPhen scores, as well as consideration of conservation and regulatory potential and full literature review. Prioritized variants were validated by Sanger sequencing in all affected individuals, with segregation extended to parental samples where available. Sequence coordinates for PTDSS1 were derived from NM_014754.1, and protein coordinates for PSS1 were derived from NP_055569.1.

Culture of fibroblasts. Skin fibroblasts from subjects 1, 4 and 5 with LMS and from three controls (healthy individuals) were grown in Ham's F12 medium supplemented with $10 \%$ FBS. Tissue culture medium and FBS were from Invitrogen. All fibroblast cultures used were negative for mycoplasma contamination.

Phosphatidylserine synthase in vitro activity. Lysates were prepared by sonication (setting 4 on a probe sonicator; $2 \times 10$ s) of cells in $10 \mathrm{mM}$ HEPES buffer ( $\mathrm{pH} 7.5$ ) containing $0.25 \mathrm{M}$ sucrose. Lysates were then centrifuged at $600 \mathrm{~g}$ for $2 \mathrm{~min}$ to pellet cellular debris and nuclei. Serine-exchange activity (contributed by both PSS1 and PSS2) was measured in the supernatant $(25-50 \mu \mathrm{g}$ of protein) in the presence of $10 \mathrm{mM}$ calcium chloride, $4 \mathrm{mM}$ hydroxylamine, $25 \mathrm{mM}$ HEPES ( $\mathrm{pH} 7.4)$ and $\mathrm{L}-\left[{ }^{3} \mathrm{H}(\mathrm{G})\right]$ serine $(50 \mu \mathrm{Ci} / \mu \mathrm{mol}$, $0.4 \mathrm{mM}$ ) (PerkinElmer) ${ }^{19}$. Assays were performed in a $200-\mu \mathrm{l}$ volume for $20 \mathrm{~min}$ at $37^{\circ} \mathrm{C}$ and were terminated by the addition of chloroform/methanol (2:1 solution). The lower phase was washed three times with methanol/water
(1:1 solution), and radioactivity was measured. For specific determination of PSS1 and PSS2 activities, choline chloride $(50 \mathrm{mM})$ was included in the assay mixture.

Metabolic labeling of phosphatidylserine and phosphatidylethanolamine. Cells were grown to $70 \%$ confluence in $100-\mathrm{mm}$ dishes, washed twice with Ham's F12 medium and incubated in the same medium to which we added $5 \mu \mathrm{Ci} / \mathrm{ml}$ of $\mathrm{L}_{-}\left[{ }^{3} \mathrm{H}(\mathrm{G})\right]$ serine (PerkinElmer) for $0,2,4$ or $6 \mathrm{~h}$. Lipids were extracted ${ }^{46}$ and separated by thin-layer chromatography in the developing solvent chloroform/methanol/acetic acid/formic acid/water (70:30:12:4:2). Phospholipids were visualized by exposure to iodine vapor, and phosphatidylserine and phosphatidylethanolamine were identified by comparison to authentic phosphatidylserine and phosphatidylethanolamine (Avanti Polar Lipids). Incorporation of radioactivity into phosphatidylserine and phosphatidylethanolamine was quantified by scintillation counting.

Measurement of phospholipid mass. Lipids were extracted from cell lysates according to the method of Bligh and Dyer ${ }^{46}$. The lower phase from the lipid extraction was dried under a stream of nitrogen gas, and lipids were separated by thin-layer chromatography as described above. The bands corresponding to phosphatidylserine, phosphatidylethanolamine and phosphatidylcholine were scraped from the plate, and the amount of phospholipid was determined by measuring the amount of inorganic phosphorus ${ }^{47}$. It is noteworthy that, in this solvent system, the phosphatidylcholine band slightly overlaps that corresponding to phosphatidylinositol.

Resistance of serine-exchange activity to inhibition by phosphatidylserine. Cells were seeded at $4 \times 10^{5}$ cells per $100-\mathrm{mm}$ dish. After $3 \mathrm{~d}$, cells were collected and suspended in buffer containing $10 \mathrm{mM}$ HEPES ( $\mathrm{pH}$ 7.5) and $0.25 \mathrm{M}$ sucrose and were then sonicated $(2 \times 10 \mathrm{~s})$ and centrifuged at $600 \mathrm{~g}$ for $2 \mathrm{~min}$ to eliminate cell debris. After cell lysates were incubated on ice for 60 min with or without phosphatidylserine liposomes, serine-exchange activity was measured in cell lysates as described above.

$\left[{ }^{3} \mathrm{H}\right]$ serine labeling in intact cells in the absence or presence of phosphatidylserine liposomes. Cells were seeded at $1.5 \times 10^{5}$ cells per $60-\mathrm{mm}$ dish. After $3 \mathrm{~d}$, cells were incubated in fresh growth medium with or without $80 \mu \mathrm{M}$ phosphatidylserine at $37^{\circ} \mathrm{C}$ for $2 \mathrm{~h}$ and were then labeled with $\left[{ }^{3} \mathrm{H}\right]$ serine for $3 \mathrm{~h}$ at $37^{\circ} \mathrm{C}$ in the corresponding growth medium with or without exogenous phosphatidylserine. Phospholipids were extracted ${ }^{46}$ and separated by thin-layer chromatography, and radioactivity was measured in phosphatidylserine and phosphatidylserine-derived phosphatidylethanolamine as described above.

RT-qPCR. Human embryonic tissues were obtained from termination of pregnancy at Queen Charlotte's and Chelsea Hospital and from the Wellcome Trust- and Medical Research Council-funded Human Developmental Biology Resource at the UCL Institute of Child Health ${ }^{48}$, as approved by the Hammersmith, Queen Charlotte's and Chelsea and Acton Hospital Research Ethics Committee (project registrations 2001/6029 and 2001/6028) and the Joint University College London/University College Hospital Committee on the Ethics of Human Research, respectively. Total RNA was isolated from fetal tissues and fibroblasts using TRIzol reagent (Invitrogen), and samples were treated with TURBO DNase (Ambion). First-strand CDNA was synthesized using up to $1 \mu \mathrm{g}$ of RNA with Moloney Murine Leukemia Virus (M-MLV) Reverse Transcriptase (Promega). RT-PCR was performed with Power SYBR Green Master Mix (Life Technologies), using StepOne Plus Real-Time PCR Systems (Applied Biosystems). The amplification reaction included an initial incubation at $50^{\circ} \mathrm{C}$ for $2 \mathrm{~min}$, polymerase activation at $95^{\circ} \mathrm{C}$ for $10 \mathrm{~min}$, and 40 cycles of denaturation at $95^{\circ} \mathrm{C}$ for $15 \mathrm{~s}$ and annealing and extension at $60{ }^{\circ} \mathrm{C}$ for $1 \mathrm{~min}$. Each RT-PCR run was subjected to melt curve analysis to confirm the fidelity of amplification of the target sequence. Resulting data were analyzed with StepOne v2.1 software. Each sample was run in triplicate, and its $\Delta C_{t}$ value was calculated using the levels of GAPDH mRNA as an endogenous control. $\Delta \Delta C_{t}$ values were calculated by $\Delta C_{t}$ (target) $-\Delta C_{t}$ (reference sample), where the reference sample consisted of five pooled fibroblast cDNAs and was included on each plate to correct for interplate variation. The relative expression of the target mRNA was calculated using the formula $2^{-\Delta \Delta C t}$. 
Zebrafish experiments. Zebrafish (Danio rerio) embryos were obtained from a wild-type strain and were raised at $28.5^{\circ} \mathrm{C}$. Microinjections $(\sim 1 \mathrm{nl})$ were performed at the one-cell stage. The expression vector used was PTDSS1 (NM_014754) Human cDNA Clone (OriGene). Mutant versions of this construct were generated using the QuikChange Site-Directed Mutagenesis kit (Stratagene). The sequences for all primers are available upon request. Capped RNA was synthesized using the mMESSAGE mMACHINE kit (Ambion). Alcian blue staining of embryos was performed using standard techniques. TUNEL staining was performed using the DeadEnd Fluorometric TUNEL System (Promega), and immunostaining for phosphorylated histone H3 was performed using standard procedures. For imaging of neural crest cells, heterozygous sox 10: $\mathrm{gfp}$ transgenic embryos were used ${ }^{49}$. All experiments with zebrafish were performed in compliance with local ethical and Home Office (UK) regulations, project license 70/6875.

Electron microscopy. Skin biopsies, buffy coat lymphocytes and fibroblast cultures were taken for ultrastructural examination. Samples were fixed in 2.5\% glutaraldehyde buffered with $0.1 \mathrm{M}$ sodium cacodylate ( $\mathrm{pH} 7.2$ ), postfixed in $1 \%$ osmium tetroxide, dehydrated in ascending grades of alcohol, processed through propylene oxide and embedded in Epon resin. Ultrathin sections were cut with a diamond knife on a Leica Ultracut UCT Ultramicrotome, placed on copper grids and stained with uranyl acetate and lead citrate. Examination was carried out with a JEOL 1400 transmission electron microscope.

Staining of neutral lipids and quantification of triacylglycerols. Control and LMS skin fibroblasts were plated at $8 \times 10^{3}$ cells $/ \mathrm{cm}^{2}$ on glass coverslips coated with poly-D-lysine (Sigma) and cultured for $48 \mathrm{~h}$ in Ham's F12 medium containing 10\% FBS. Cells were fixed with $4 \%$ paraformaldehyde for $15 \mathrm{~min}$ at room temperature and washed three times with PBS. Neutral lipids in cellular lipid droplets were stained by incubating cells with BODIPY 493/503 (Molecular Probes; $2 \mu \mathrm{g} / \mathrm{ml}$ in PBS) for $15 \mathrm{~min}$ at room temperature, and cells were then washed three times. Nuclei were stained with $100 \mathrm{nM}$ 4',6'-diamidino-2-phenylindole dihydrochloride (DAPI; Molecular Probes), and cells were mounted on slides using ProlongGold (Molecular Probes). Images were acquired with a spinning-disk Olympus-IX81 confocal microscope and analyzed with Volocity software (PerkinElmer). Fluorescence was quantified using the same software. Cellular triacylglycerols were quantified using the Trig/GB kit (Roche) according to the manufacturer's instructions.

Quantitative analysis of Annexin V-positive cells. Skin fibroblasts from three controls and three individuals with LMS (subjects 1, 4 and 5) were plated at $2 \times 10^{4}$ cells/well in a 24 -well plate and incubated for $48 \mathrm{~h}$ in $500 \mu \mathrm{l}$ of DMEM supplemented with $10 \% \mathrm{FBS}\left(5 \% \mathrm{CO}_{2}\right.$ and $\left.37^{\circ} \mathrm{C}\right)$. After cells were gently washed with cold PBS, $500 \mu 1$ of Annexin binding buffer (10 mM HEPES, $140 \mathrm{mM} \mathrm{NaCl}, 2.5 \mathrm{mM} \mathrm{CaCl}_{2}, \mathrm{pH} 7.4$ ) containing $1 \mu \mathrm{l}$ of Annexin V-Alexa Fluor 568 conjugate (Life Technologies, Molecular Probes; 1:500 dilution) and $1 \mu \mathrm{l}$ of Hoechst (Life Technologies, Molecular Probes; 1:500 dilution) was added to each well, and cells were incubated for $45 \mathrm{~min}$ at room temperature. Cells were washed again with Annexin binding buffer and fixed with $4 \%$ paraformaldehyde for $15 \mathrm{~min}$. Cells were washed three more times to remove excess paraformaldehyde and were immersed in Annexin binding buffer for analysis. Images were acquired using an Olympus IX70 inverted fluorescent microscope. Five random microscopic fields were analyzed for each well. Numbers of total cells (Hoechst positive) and Annexin V-positive cells were counted. Experiments were performed in triplicate. Student's $t$ test was used for statistical analysis.

Other methods. Immunoblotting was performed using polyclonal antibody to PTDSS1 (Y-19, sc-51410, Santa Cruz Biotechnology) ${ }^{27}$ and standard methods. Cellular protein content was determined by the BCA method (Pierce) with BSA as the standard. Statistically significant differences between samples were assessed by the two-tailed Student's $t$ test. $P$ values of $<0.05$ were considered to be statistically significant.

46. Bligh, E.G. \& Dyer, W.J. A rapid method of total lipid extraction and purification Can. J. Biochem. Physiol. 37, 911-917 (1959).

47. Rouser, G., Siakotos, A.N. \& Fleischer, S. Quantitative analysis of phospholipids by thin-layer chromatography and phosphorus analysis of spots. Lipids 1, 85-86 (1966).

48. Lindsay, S. \& Copp, A.J. MRC-Wellcome Trust Human Developmental Biology Resource: enabling studies of human developmental gene expression. Trends Genet. 21, 586-590 (2005).

49. Carney, T.J. et al. A direct role for Sox 10 in specification of neural crest-derived sensory neurons. Development 133, 4619-4630 (2006). 